Boise State University

ScholarWorks

6-1-2013

Using Balanced Learning Course Design to Reduce Resistance to Diversity Curricula

Misty L. Wall

Boise State University 


\title{
Using Balanced Learning Course Design to Reduce Resistance to Diversity Curricula
}

\author{
Misty L. Wall \\ School of Social Work, Boise State University, Boise, Idaho, USA
}

Corresponding author:

Misty L. Wall, PhD, MSSW, LCSW, Assistant Professor, School of Social Work, Boise State University, 1910

University Drive, Boise, ID 83725-1940, USA

Email: mistywall@boisestate.edu, Phone: +00 1208426 1711, Fax: +00 12084264291

\begin{abstract}
Diversity content is often met with covert and overt resistance in the classroom. Utilisation of a balanced learning model may address classroom resistance. Balanced learning models require 'backwards' course planning, equal attention to course content and class process, and a strategic use of cyclical course design to include introduction of new materiel, experience, followed by personal reflection. Using a balanced learning model moves classroom instruction beyond active learning strategies in the classroom, but can present unique challenges for instructors. While not without challenges, utilising a balanced learning model proves useful during course design and delivery of a graduate course on diversity. The article describes balanced learning models emergence, define the benefits of such models, and ultimately demonstrate how using a balance learning model is a useful course design tool to reduce resistance to diversity content in the classroom.
\end{abstract}

Keywords: education, diversity, course design, challenges, balanced learning model

\section{Introduction}

For decades talented instructors and scholars have grappled with identifying successful methods of infusing diversity content in a way that creates significant and sustained learning (Tatum 1992, 1997, Van Soest 1994), while creating a cohesive and safe classroom experience. While no single method has been identified a that guarantees a pleasant and/or meaningful classroom experience, several have contributed valuable techniques and ideas to addressing the challenge of delivering diversity content. It is the argument of this author that using a balanced course design, as articulated by Fink (2003), is an effective method of addressing common challenges in delivering diversity content while creating a significant learning experience for students. Fink's work is especially fitting because his model expands on existing active learning literature to include a more holistic approach to learning. Specifically, Fink includes planned and essential reflection as a part of the model (rather than simply seeing and doing) and emphasises how each component (information and ideas, experience, and reflection) is inherently related. The purpose of this article is to describe Fink's model of balanced course design and demonstrate the value of using such a design when planning a diversity course. This aim will be achieved by providing an 
overview of the challenges associated with delivering diversity curricula and the emergence of Fink's (2003) model of balanced learning, and by exploring the use of Fink's model during the design of a graduate level diversity course.

\section{Literature review}

The following provides a brief overview of challenges associated with delivering diversity curricula, which is an essential concern for social work and other helping professions. Next, an exploration of active learning theories and models which eventually gave rise to Fink's (2003) expansion of active learning to include the importance of utilising a balanced learning model in course design as a strategy to address and reduce the challenges linked to delivery of diversity content.

\section{Challenges in delivering diversity content}

The Council on Social Work Education (CSWE) began prescribing inclusion of specific content on diverse populations in the late 1960s when they called for specific content on women and people of colour to be included in every accredited social work programme so that emerging social work practitioners would be equipped to work in a diverse society. As population demographics have changed, and new marginalised populations become evident, CSWE has continued to expand the expectation of social work education to add content relevant to oppression and marginalisation of all groups based on colour, religion, ethnicity, gender, and sexual orientation (Council on Social Work Education [CSWE] 1992).

The CSWE expanding the focus of diversity to include the experience of oppression and marginalisation of minority groups, rather than content on the groups themselves, is an important departure from the original call in the 1960s requiring content be added about women and people of colour. Accurate content about diverse populations is essential, but content alone is insufficient to ensure the affective and behavioural shifts necessary to practice effectively with minority groups (Gutierrez et al. 1999). Meaningful learning about cultures and diversity results in changes in cognition, affective processes, and behavioural changes (Heuberger et al. 1999) such as understanding how attitudes can lead to bias and have negative impacts on service outcomes (Greene 1994, Gutierrez et al. 1999). This shift from content delivery to facilitating affective and behavioural changes is in line with contemporary learning theories that suggest classrooms should be focused on change in the learner, and define significant learning as a lasting change that impacts all aspects of the learner's life. In summary, true learning can be defined through affective and behavioural shifts, course work is the catalyst for this shift, and course design has to be approached with these shifts in mind. Approaching course work designed to challenge student's thoughts, feelings, and behaviours is bound to have challenges.

Faculty have continuously found delivering diversity content is complex and can be the nexus for negative classroom experiences for both teacher and student. Common complaints from student's taking diversity courses or facing diversity content include instructor's lack of introspective and emotional preparation (Miller et al. 2004), and over focus on content to the detriment of attention to classroom emotion and the inevitable tensions that coincide with diversity content (Mishna \& Bogo 2007). Often instructors observe student resistance to course material (Schmitz et al. 2001, Abrams \& Gibson 2007), students' challenging instructor legitimacy to teach diversity or diverse content (Miller et al. 2004), dissatisfaction with instructor response to student needs (Lewis 1993, Garcia \& Van Soest 1999, Miller et al. 2004). Instructors and students alike report difficult classroom experiences when the content is diversity, this may be because the content requires personal introspection often leading to affective and behavioural shifts, which can be 
uncomfortable. Utilising balanced course design is one strategy instructors can use to reduce resistance to diversity content.

\section{Active learning and balanced course design}

Active learning theorists suggest active learning or learning-centred teaching should be guided by the goal that the classroom provides carefully crafted experiences, leading to significant learning experiences, which then lead to behavioural changes. The focus on behavioural changes make these theories especially relevant to the desired outcomes associated with including diversity content in social work and other helping professions classrooms. Learning theorists, such as L. Dee Fink, have expanded on traditional active learning theories to introduce the idea that simply adding components that require action, such as internships, in-class exercises or discussion, service learning, or practicum hours without changing the structure and objectives of the course, may not be adequate to facilitate lasting learning (Fink 2003). He writes:

"In a powerful learning experience, students will be engaged in their own learning, there will be a high energy level associated with it, and the whole process will have important outcomes or results. Not only will students be learning through the entire course, by the end of the course they will clearly have changed in some important way - they will have learned something important. And that learning will have the potential for changing their lives." (Fink 2003, p6)

Rather, including a balance of content delivery (information), experience (activity), in addition of time for reflection through reflective dialogue can benefit the student more than information and activity alone (see Figure 1). This is a significant contribution to literature on learning because it recognises the significance and vital importance of moving beyond the see-do-learn models of active learning to include reflective dialogue during which time

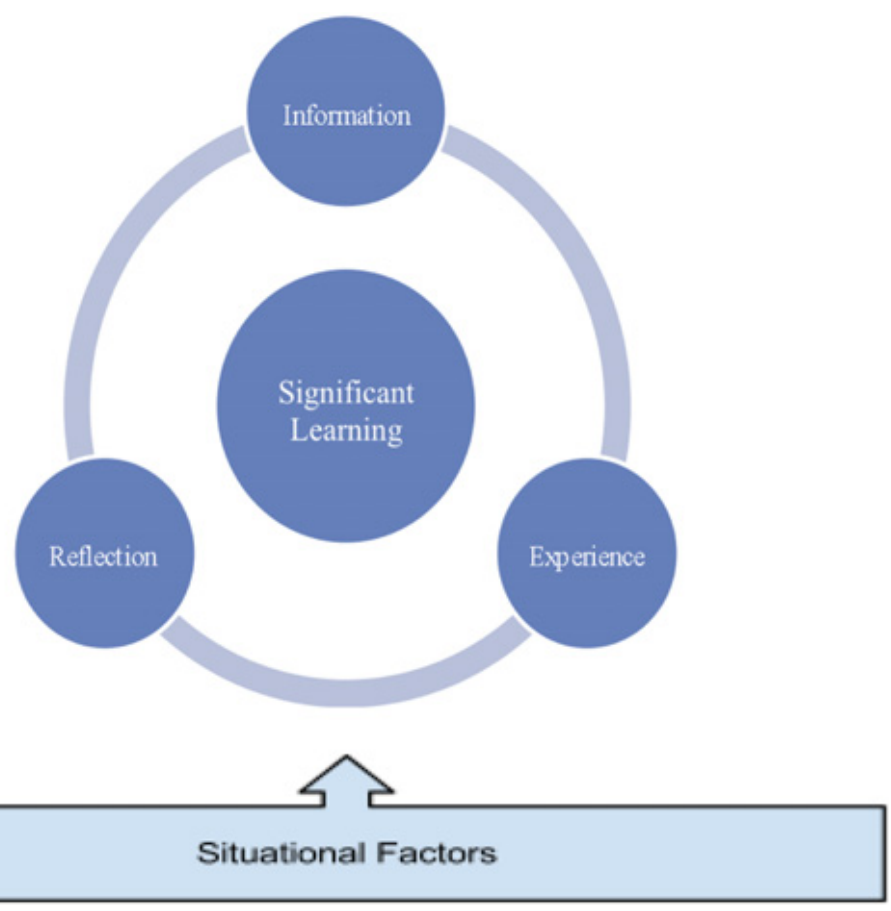

Figure 1 Balanced learning paradigm 
students make sense of information and experiences thus making them relevant to practice after graduation.

\section{Implementing balanced course design}

\section{Course logistics}

The course, Advanced Topics in Human Diversity, was created as a mandatory bridge course for incoming Advanced Standing students in a graduate school of social work. The course was designed to addressed an identified need for increased knowledge and tolerance of diverse populations among incoming graduate students (Wall \& Rainford 2013). Using Fink's model of balanced design the course was highly experiential, requiring overt and candid investigation of personal identity development. Course educational objectives included: (1) demonstration of commitment to seeking strengths in all forms of human diversity; (2) articulation of student's own identity development process and its implication for social work practice; and (3) demonstration of commitment to critical self-assessment and reflective practice. The class met twice a week, three hours per class, over eight weeks. The eight weeks were divided into three distinct learning modules, and each course session was designed to facilitate significant learning using the balanced model previously discussed.

\section{Creating safety}

The idea of creating safety in the social work classroom is necessary for students to explore and challenge their own values, experiences, and worldview; and conversely to hear the values and worldviews of others (Chan \& Treacy 1996, Garcia \& Van Soest 1999, Holley \& Steiner 2005). Further, students rely on the instructor to create and maintain a sense of safety in the classroom (Hyde \& Ruth 2002). Strategies to creating safety in the classroom are abundant in social work literature (see Mishna \& Bogo 2007) and many were used during the duration of the course Advanced Topics in Human Diversity. However, a full review of in-class strategies to create a sense of safety is beyond the scope of this article, thus two primary strategies from the literature regarding course design (rather than classroom management) are discussed next.

\section{Use of learning teams}

Using small groups is a common teaching strategy used within active learning and balanced learning models. Small groups, or learning teams, can be the catalyst for powerful learning on many levels. Thoughtful use of small groups or learning teams can facilitate learning about content, systems, cross-cultural communication, working with others, appreciating the empathetic experiences of others, and self as a vehicle for change (Michaelsen et al. 2002, Fink 2003). The teams for Advanced Topics in Human Diversity were created by the instructor based on information gathered during initial class discussion including length of time in practice, type of practice, life responsibilities outside of graduate school, life span, etc. Five teams were created, four groups had five people and one had six. Teams were established on the second night of class, remained consistent throughout the semester, and classroom logistics allowed them to sit at team tables during in-class sessions, but they were manipulated during larger class discussions. Two community visits occurred in Teams, timeline and crest projects were shared with teams. Peer accountability was established by having peer evaluations of team member contributions at midterm and the end of the course, peer evaluation (using instructor led, but class created, rubrics) of projects, and anonymous narrative feedback at various points during the course (after particularly heated class discussion or controversial speakers). The instructor of the course provided individual feedback based on class-established guidelines for participation twice 
during the semester (once during week three and once during week six) via the blackboard. Additionally, a survey was distributed via the blackboard at midterm to the entire class (large team) to evaluate progress on class guidelines and goals. Results of the class survey were shared (anonymously) via the blackboard and discussed during class.

\section{Learning modules}

The first learning module was roughly three weeks (18 hours, over six sessions) and centred on discovery of self. The second learning module expanded newly gained, or articulated, self-awareness to include rich, qualitative experiences with various marginalised and vulnerable groups (18 hours, over six sessions). During the second module, weekly curricula included structured topics including, but not limited to, classism, racism, abelism, sexism, heterosexism, and religious tolerance. Finally, the third module (12 hours, over four sessions) consisted of reflection on the adjusted self, and through reflection by peers a new cycle of information, experience, and reflection.

\section{Module One: self-discovery}

Awareness of self is an essential aspect of successful and ethical social work practice and is a central concept in social work education and an ongoing requirement for professional practice. The National Association of Social Workers (NASW) created guidelines for culturally component practice that specifically included self-awareness as a requirement for effectively working with diverse populations calling social workers to understand their own personal, cultural values and beliefs as a way to appreciate the importance of these aspects of identity in others (National Association of Social Workers [NASW] 2000). Additionally, the Council on Social Work Education (CSWE) requires students to successfully demonstrate self-awareness as a core competency in an effort to minimise the influence of personal biases and values in working with people (Council on Social Work Education [CSWE] 2008). Being aware of personal identity, values, biases, and sense of self is essential competent practice. The purpose of including content on self-discovery serves to provide students time to explore the origins and current aspects of their identity that will inevitably impact the way they interact with others and provide client services.

The primary assignment during Module One was the completion of a personal timeline. Each student was required to prepare a timeline of significant events in his or her life from a bio-psycho-social-cultural-spiritual perspective. The timeline could take any shape the student desired, but specific grading requirements were provided to students during the first class session. A secondary assignment during Module One was presentation of the complete timeline to their team. Peer evaluation of the timeline and presentation of the timeline were utilised to encourage accountability. The instructor gathered peer evaluations and distributed them anonymously to each student. Despite the linear project label "timeline", the completed projects were as varied in form as are students. Some of the most memorable included a hand-painted skateboard used by the student daily to overcome taking herself and her age too seriously, a display of more than 40 shoes in various styles correlated to the student's experiences ranging from dirty work boots to leopard printed stiletto heels, and a mobile wardrobe including clothing for every year of life telling the story of a transgender man.

\section{Module Two: experience}

The second module focused on creating experiential experiences for students to have positive encounters with people and places with which they were unfamiliar. There were four types of experiences during the course. The first was Class Community Visits where the entire class visited a location selected by the instructor. By experiencing the newness of suddenly being unaware of the group norms, students were thrust into the position of 
minority, and through reflection and discussion were able to connect their feelings of the minority to gain empathy and appreciation for differences. While creating a learning experience that stimulates personal reflection and new awareness of self is pivotal, Community Visits also stimulate skills and desire for lifelong curiosity and learning.

The second experience type was Team Community Visits where students worked in their teams to select and visit two community locations that serve minority populations. Following the Team Community Visits students were required to do one Community Visit alone. Finally, various in-class experiential events were scheduled, such as panel presentations, individual presentations, movies, music, etc.

\section{Community visits}

Hare Krishna temple. The second Class Community Visit was to the local Hare Krishna temple for a feast and celebration of their Guru Gopel Goswami Krishna's annual visit from India. During the visit to the Krishna temple we participated in a traditional Krishna celebration beginning with chanting dancing, singing followed by a short talk by the Guru, an open question and answer session with the Guru, then a traditional Hare Krishna vegetarian feast and socialising with Krishna devotees and visitors. Visiting the Hare Krishna temple offered students the chance to actively participate as a new member of a group with unfamiliar rituals, customs, rules, and expectations.

Tae kwon do studio visit. The entire class visited a local tae kwon do studio where students participated in a beginning level martial arts course followed by a panel presentation by the tae kwon do three masters who were born and raised in South Korea two of whom had been in the US for seven years, the other having been in the US for one year.

Team community visits. Student teams (usually five people) were required to visit two community sites that serve vulnerable persons or minority groups. The sites could be anywhere and serve any population however the chosen site had to be somewhere no one in the team had visited before. Students were encouraged to expand their perception of minority groups beyond social service agencies to include places that people want to be, such as the LGBTOIA (lesbian, gay, bisexual, transgender, queer/questioning, intersex, ally) Community Centre, ethnic grocery stores, an Islamic mosque, etc. Students were required to communicate with at least one person at the Community Visit site and the visit was expected to last at least two hours (excluding travel time). Community visits were initially conducted in Teams so that students were able to experience new cultures and experiences that often generate discomfort but had support from team mates which kept discomfort from elevating to a level that prevented learning. Students were required to participate in Team Community Visits within the second module, which fell within the fourth to sixth weeks of class so that visits occurred after the self-discovery module and timeline projects were complete.

In-class experiences. During the semester three sessions were devoted to sharing information about identify development such as Helms' (1990) model of White identity development and Cass's (1984) model of gay and lesbian identity development. These models were presented as a classic theoretical backdrop, and provided students with a theoretical springboard from which they could begin to assemble, or disassemble, the pieces that made up their identity. Following the in-class module on identity development, the class was visited by a panel of men and women from various racial backgrounds. We viewed the movie The Color of Fear and engaged in an, often heated, discussion about race. During the gender and sexism class I utilised music to spark conversation. The National Alliance on Mental IIIness (NAMI) provided three speakers to provide first-hand accounts of living with mental illness to address the topic of abelism, and a panel of religious leaders joined the class to discuss religious tolerance. 


\section{Module Three: reflection}

Reflection in practice is essential. Reflection following practice allows the clinician to assimilate new learning/understanding to each given situation (Mishna \& Bogo 2007) and is often defined as the deliberate action of reviewing one's work after the event to determine factors that affected the outcome in order to learn (change) practice (Cross et al. 2004, Bogo 2006). In short, reflection is where learning is made relevant and 'work' occurs through the class experiences with the goal of changing practitioner behaviour (learning). Following all experiential activities students were required to reflect using a guided journal format that includes addressing the cognitive, affective, and behavioural responses prior to and after the event. Focus on the three dimension of reflection (cognitive, affective, and behavioural) is stressed in experiential-based learning (Welch 1999, Bringle \& Hatcher 2002) because within the assumptions of team based learning (TBL) and balanced course design learning is considered to be more than solely a cognitive endeavour and meaningful learning about cultures and diversity will stimulate changes in cognition, affective processes, and behaviour (Heuberger et al. 1999). After thoughts were recorded in writing, the were shared with team mates, followed by classroom discussion, as is common with the TBL framework utilised.

\section{Discussion}

I began this project with the hope of understanding the level of homophobia and heterosexism among incoming gradate students in our rural, Pacific northwest university and to determine the effectiveness of applying a balanced course design in reducing such attitudes. It appears that the balanced course design allowed for a successful class experience, with little student or classroom resistance. Students provided feedback that the teams were instrumental in tackling sensitive information, classroom and class visits successfully generated empathy for minority groups and behavioural changes, and assignments required deep introspective into personal and professional patterns, beliefs, and values. The following provides a brief account of the impact that the combination of information, experience, and reflection had on generating significant learning.

Feedback about the teams was positive with many students reporting that the teams made them prepare for class in a way that was unusual for them because they wanted their classmates to be 'proud' of their work. Using teams encouraged some students to share when that was generally outside of their comfort zone, and during community visits encouraged them to take risks that they would generally avoid (such as eating tongue).

Reflection and discussion regarding visiting the Temple was very positive. Cognitively, students articulated the difference in time management of their faith experiences, which were primarily traditional Christian, Catholic, or Mormon. They were surprised at the 'free flowing' nature of worship, noting that people seemed to come and go, openly asking probing and at times challenging questions to the Guru, some danced while others remained seated, the high activity level and participation from the children, among other things. Many students discussed the emotions evoked during a ceremony that paid homage to many idols rather than a single god, and the behavioural choices they made in relation to such strong emotions. Students were successfully able to compare and contract these experiences with their own, and articulate appreciation for both.

Feedback about participating in tae kwon do was also very positive, although both Community Visit locations are welcoming, students were able to articulate the difference in structure, formality, and behavioural expectations between the two. More importantly, student were able to identify strengths in both Community Visit locations and groups, and ways that the new experiences could be integrated into their personal and professional life. 
For instance, one student initiated a powerful conversation about race, conformity, and intersectionality generated from the requirement to address the tae kwon do leaders as 'Master' and the cognitive, affective, and behavioural chaos this brought up for him as a Caucasian man raising an African American son.

One of the most powerful reflections regarding Community Visits was caused by a team visit to an Islamic mosque where the team walked into the front door and was immediately separated when the women were rushed out of the front door and expected to enter in the back of the building through a service entrance owing to the cultural tradition of men and women worshipping separately. This experience was equally powerful for all team members, but was perceived differently by each. For instance, one woman who was a devout member of a faith that actively works to eliminate gender inequality was very disturbed and said that she felt like she had, for the first time, felt mistreated, 'inferior' and 'dirty' because she was a woman. Another man in the team who identifies as a 'feminist' wrote in his journal that consciously staying with the men, mostly out of fear of confrontation with a seemingly adamant group of men, a culture that was unknown to him, and fear of losing a good grade in the class, was one of the most shameful things he had ever done. Later he wrote that it took "very little force and about 15 seconds for me to justify sexism for my Grade Point Average". This student's comments drew clear parallels to the impact of all three dimensions of reflection as he made sense of this experience and planned for behavioural differences in the future.

\section{Conclusion}

Commitment to ensuring diversity content is included in social work education has been well documented. The manner in which diversity content is included is less consistent and varies from being infused across all courses, or included within a specific course. Moreover, the benefits of graduating social workers with a strong grasp of diverse populations to clients are certainly plentiful. Yet, how to ensure students are graduating with desired affective and behavioural responses is uncertain, and research points to student resistance including emotional withdrawal, questioning the instructor, even feeling unsafe or unprotected by the instructor. Within this article these challenges were reviewed, along with the introduction of using balanced course design as a method that may address many of these negative classroom experiences and aim at generating not only positive classroom experiences but also, more importantly, lasting and deep learning. Balanced learning, an update to active learning models, balances focus equally between class content and class process which reduces resistance to diversity content and creates significant learning opportunities for students to experience (rather than apply) new concepts. Balanced course design leads to increased knowledge, understanding, and empathy for diverse individuals and groups. In the balanced learning model, information and experience are followed by critical, personal reflection allowing students to sort out new information and experiences in a way that makes them individually meaningful. Future research that is longitudinal in nature and is aimed at exploring how and when students incorporate classroom learning into practice is needed. This method of course design was utilised during design and implementation of a graduate level social work course, designed to address Advanced Standing students entering a rural programme with little experience, knowledge, and empathy with diverse populations.

\section{References}

Abrams, L.S. and Gibson, P. (2007) Reframing multicultural education: teaching white privilege in the social work curriculum. Journal of Social Work Education 43 (1), 147-160. 
Bogo, M. (2006) Social work practice: concepts, processes, and interviewing. New York: Columbia University Press.

Bringle, R.G. and Hatcher, J.A. (2002) Campus-community partnerships: the terms of engagement. Journal of Social Issues 58 (3), 503-516.

Cass, V. (1984) Homosexual identity formation: testing a theoretical model. Journal of Sex Research 20 (2), 143-167.

Chan, C.S. and Treacy, M.J. (1996) Resistance in multicultural courses. American Behavioral Scientist 40, 212-221.

Council on Social Work Education [CSWE] (1992) Educational policy and accreditation standards. Retrieved from http://www.cswe.org.

Council on Social Work Education [CSWE] (2008) Educational policy and accreditation standards. Retrieved from http://www.cswe.org.

Cross, V., Liles, C., Conduit, J. and Price, J. (2004) Linking reflective practice to evidence of competence. Reflective Practice 5 (1), 3-31.

Fink, L.D. (2003) Creating significant learning experiences: an integrated approach to designing college courses. San Francisco, CA: Jossey-Bass.

Garcia, B. and Van Soest, D. (1999) Changing perceptions of diversity and oppression: MSW students discuss the effects of a required course. Journal of Social Work Education 33 (1), 119-129.

Greene, B. (1994) Ethnic-minority lesbians and gay men: mental health and treatment issues. Journal of Consulting and Clinical Psychology 62 (2), 243-251.

Gutierrez, L., Fredricksen, K. and Soifer, S. (1999) Perspectives of social work faculty on diversity and societal oppression content: results from a national survey. Journal of Social Work Education 35, 409-419.

Helms, J. (1990) Black and White racial identity: theory, research, and practice.

Contributions in Afro-American and African studies. New York, NY: Greenwood Press.

Heuberger, B., Gerber, D. and Anderson, R. (1999) Strength through cultural diversity. College Teaching 47 (3), 107-116.

Holley, L. C. and Steiner, S. (2005) Safe space: student perspectives on classroom environment. Journal of Social Work Education 41 (1), 49.

Hyde, C.A. and Ruth, B.J. (2002) Multicultural content and class participation: do students self-censor? Journal of Social Work Education $\infty, 241-256$.

Lewis, E.A. (1993) Continuing the legacy: on the importance of praxis in the education of social work students and teachers. In Multicultural teaching in the university (eds.

D. Schoem, L. Frenkel, X. Zuniga and E.A. Lewis). Westport, CT: Praeger, pp26-36.

Michaelsen, L., Knight, A. and Fink, D. (2002) Team based learning. Westport, CT: Begin \& Garvey.

Miller, J., Hyde, C.A. and Ruth, B.J. (2004) Teaching about race and racism in social work: challenges for White educators. Smith College Studies in Social Work 74 (2), 409-426.

Available from ProQuest Psychology Journals (document ID 621036381) (retrieved 17 July 2009).

Mishna, F. and Bogo, M. (2007) Reflective practice in contemporary social work classrooms. Journal of Social Work Education 43 (3), 529-541. Available from ProQuest Psychology Journals (document ID 1425097161) (retrieved 17 July 2009). 
National Association of Social Workers [NASW] (2000). NASW code of ethics. Washington, DC: NASW.

Schmitz, C.L., Stakeman, C. and Sisneros, J. (2001) Educating professionals for practice in a multicultural society: understanding oppression and valuing diversity. Families in Society: The Journal of Contemporary Human Services 82 (6), 612-622.

Tatum, B.D. (1992) Talking about race, learning about racism: the application of racial identity development theory in the classroom. Harvard Educational Review 62 (1), 1-24.

Tatum, B.D. (1997) Why are all the Black kids sitting in the cafeteria and other conversations about race. New York: Basic Books.

Van Soest, D. (1994) Social work education for multicultural practice and social justice advocacy: a field study of how students experience the learning process. Journal of Multicultural Social Work 3 (1), 17-28.

Wall, M. L. and Rainford, W. (2013) Testing the "learning journey" of MSW students in a rural program. Journal of Teaching in Social Work 33 (1), 75-86.

Welch, M. (1999) ABCs of reflection. NSEE Quarterly 25 (1), 22-25. 